\title{
Automated external cardioversion defibrillation monitoring in cardiac arrest: a randomized trial Bakhtiar Ali1 ${ }^{1}$, Heather Bloom ${ }^{1,2}$, Emir Veledar ${ }^{2}$, Dorothy House ${ }^{1}$, Robert Norvel ${ }^{1}$, Samuel C Dudley ${ }^{3}$ and A Maziar Zafari*1,2
}

\author{
Address: ${ }^{1}$ Atlanta Veterans Affairs Medical Center, Decatur, Georgia, USA, ${ }^{2}$ Emory University School of Medicine, Division of Cardiology, Atlanta, \\ Georgia, USA and ${ }^{3}$ University of Illinois at Chicago, Section of Cardiology and the Jesse Brown VA Medical Center, Chicago, Illinois, USA \\ Email: Bakhtiar Ali - bakhtiar02@yahoo.com; Heather Bloom - hbloom@emory.edu; Emir Veledar - eveleda@emory.edu; \\ Dorothy House - Dorothy.House2@va.gov; Robert Norvel - rnorvel@hotmail.com; Samuel C Dudley - scdudley@uic.edu; A \\ Maziar Zafari* - azafari@emory.edu \\ * Corresponding author
}

Published: II June 2008

Trials 2008, 9:36 doi:10.1186/1745-62/5-9-36

This article is available from: http://www.trialsjournal.com/content/9/I/36

(c) 2008 Ali et al; licensee BioMed Central Ltd.

This is an Open Access article distributed under the terms of the Creative Commons Attribution License (http://creativecommons.org/licenses/by/2.0), which permits unrestricted use, distribution, and reproduction in any medium, provided the original work is properly cited.

\begin{abstract}
Background: In-hospital cardiac arrest has a poor prognosis despite active electrocardiography monitoring. The initial rhythm of approximately $25 \%$ of in-hospital cardiopulmonary resuscitation (CPR) events is pulseless ventricular tachycardia/ventricular fibrillation (VT/VF). Early defibrillation is an independent predictor of survival in CPR events caused by VT/VF. The automated external cardioverter defibrillator (AECD) is a device attached by pads to the chest wall that monitors, detects, and within seconds, automatically delivers electric countershock to an appropriate tachyarrhythmia.
\end{abstract}

Study Objectives: - To evaluate safety of AECD monitoring in hospitalized patients.

- To evaluate whether AECDs provide earlier defibrillation than hospital code teams.

Methods: The study is a prospective trial randomizing patients admitted to the telemetry ward to standard CPR (code team) or standard CPR plus AECD monitoring (PowerHeart CRM). The AECD is programmed to deliver one $150 \mathrm{~J}$ biphasic shock to patients in sustained VT/VF. Data is collected using the Utstein criteria for cardiac arrest. The primary endpoint is time-to-defibrillation; secondary outcomes include neurological status and survival to discharge, with 3-year follow-up.

Results: To date, 192 patients have been recruited in the time period between 10/10/2006 to 7/20/2007. A total of 3,655 hours of telemetry data have been analyzed in the AECD arm. The AECD has monitored ambulatory telemetry patients in sinus rhythm, sinus tachycardia, supraventricular tachycardia, atrial flutter or fibrillation, with premature ventricular complexes and non-sustained VT without delivery of inappropriate shocks. One patient experienced sustained VT during AECD monitoring, who was successfully defibrillated (I7 seconds after meeting programmed criteria). There are no events to report in the control arm. The patient survived the event without neurological complications. During the same time period, mean time to shock for VT/VF cardiac arrest occurring outside the telemetry ward was $230 \pm 50$ seconds.

Conclusion: AECD monitoring is safe and likely results in earlier defibrillation than standard telemetry monitoring.

Trial Registration: National Institutes of Health registration ID: NCT00382928 


\section{Background}

In-hospital cardiac arrest has a poor prognosis despite active electrocardiography monitoring. Part of the poor prognosis may be explained by slow response to a lethal arrhythmia.[1] The National Registry of Cardiopulmonary Resuscitation (NRCPR) has reported delayed defibrillation, which was defined as greater than 2 minutes, in more than $30 \%$ of cases of in-hospital cardiac arrest. This delay in defibrillation resulted in significantly lower probability of surviving to hospital discharge.[1]

The initial rhythm in about $25 \%$ of in-hospital cardiac arrest is ventricular tachycardia or fibrillation (VT/VF).[2] Early provision of good quality CPR and rapid defibrillation have the highest impact on survival for the victims of VT/VF cardiac arrest.[3] Early defibrillation is an independent predictor of survival in CPR events caused by VT/ VF. $[4,5]$ Delay in provision of defibrillation for $10 \mathrm{~min}$ utes renders CPR ineffective.[4] Each minute of delay in defibrillation increases the likelihood of death by $7 \%$ to $10 \%$ in cardiac arrest.[5] If defibrillation is provided within 3 minutes in in-hospital cardiac arrest, 38\% survival to discharge is reported versus $21 \%$, if defibrillation is provided after 3 minutes.[2] Addressing this delay, a program encouraging early defibrillation using automated external defibrillators (AED) in the hospital resulted in a 14-fold increase in survival for VT/VF cardiac arrest.[6]

Automated External Cardioverter Defibrillator (AECD; The PowerHeart CRM, Cardiac Science Inc., Seattle, WA) is a device attached to the chest wall by pads, monitors the electrocardiogram, and is capable of automatically delivering electric countershock to appropriate rhythms without operator intervention. Automated external cardioverter defibrillators have been studied in a few nonrandomized clinical trials. [7-9] They performed with a sensitivity of $100 \%$ and a specificity of $98.8 \%$ in a study conducted in the United States $(\mathrm{n}=79)$ and a sensitivity of $100 \%$ and a specificity of $97.6 \%$ in an European study $(\mathrm{n}=117) \cdot[7,8]$ We designed the current randomized trial to test the safety of AECDs in hospitalized patients and the performance compared to standard telemetry response.

\section{Methods}

This study is a single center, randomized, prospective, trial in which, all patients admitted to the emergency department and telemetry unit of the Atlanta Veterans Affairs Medical Center (AVAMC), Decatur, Georgia are screened. Patient recruitment started in October 2006. Inclusion and exclusion criteria are presented in Table 1. Patients consenting to participate in the study are randomized to either standard electrocardiographic telemetry or standard

Table I: Inclusion and Exclusion Criteria

\section{Inclusion Criteria:}

I. All patients admitted to telemetry ward and ER.

2. Age $>18$ years.

\section{Exclusion Criteria:}

I. Pregnant women.

2. Patients with $\mathrm{R}$ wave less than $0.5 \mathrm{mV}$ in lead II.

3. Patients with functioning ICDs.

4. Patients with cardiac pacemakers if oversensing by AECD is demonstrated (double counting of pacer spikes).

5. Patients with visible chest lesions that would prevent AECD pad placement.

6. Patients who are designated DNR.

7. Right bundle branch block.

8. Patients with Parkinson's disease.

9. Patients with seizure disorders.

\section{Additional Exclusion Criteria for Emergency Room:}

10. Patients with dementia and/or delirium.

I I. Patients presenting with psychiatric complaints.

12. Patients who are agitated.

13. Patients presenting with trauma.

14. Patients unable to participate in the informed consent process.

15. Patients with respiratory rate greater than twenty.

16. Patients who report pain greater than four out of ten in the visual analog scale.

$E R=$ emergency room; $I C D=$ internal cardioverter defibrillator; $A E C D=$ automated external cardioverter defibrillator; $D N R=$ do not resuscitate 
Table 2: Cerebral Performance Categories/CPC scale

CPC I: Good cerebral performance - conscious, alert, able to work, might have mild neurologic or psychological deficit.

CPC 2: Moderate cerebral disability - conscious, sufficient cerebral function for independent activities of daily life. Able to work in sheltered environment.

CPC 3: Severe cerebral disability - conscious, dependent on others for daily support because of impaired brain function. Ranges from ambulatory state to severe dementia or paralysis.

CPC 4: Coma or vegetative state - any degree of coma without the presence of all brain death criteria. Unawareness, even if appears awake (vegetative state) without interaction with environment; may have spontaneous eye opening and sleep/awake cycles. Cerebral unresponsiveness.

CPC 5: Brain death - apnea, areflexia, EEG silence, etc.

$\mathrm{EEG}=$ electroencephalogram

telemetry augmented by AECD monitoring (Figure 1). Patients randomized to AECD monitoring have the AECD attached for the duration of their hospitalization while undergoing simultaneous telemetry monitoring. A log book is used to record the time of AECD attachment and to record whenever the AECD is detached from the patient. The AECD is programmed to deliver a single 150 Joule shock to VT/VF rhythms presenting above the rate of 170 beats per minute, after a 30 second delay. Study enrollment terminates when telemetry is discontinued. The primary endpoint is time-to-defibrillation in VT/VF cardiac arrest. The AECDs have the ability to measure the precise time of initiation of cardiac arrhythmias and of delivery of defibrillation. The secondary outcomes are survival to discharge and cerebral outcomes as measured by cerebral performance category scale (Table 2). Patients who survive cardiac arrest while participating in the trial will be followed for a period of three years for survival, internal cardioverter defibrillator (ICD) placement and cerebral performance. The Emory University Institutional Review Board (IRB) and the AVAMC Research and Development Committee approved the study.

\section{Data collection}

Demographic and arrest data is collected according to Utstein guidelines and stored in a database designed with Microsoft access software (Microsoft Inc., Redmond, WA).

\section{Sample size}

A total sample size of 40 patients per group will be recruited. There are approximately 40 true cardiac arrests per year on the telemetry ward. The expected time to defibrillation in the AECD group is to be $30 \pm 30$ seconds, whereas time to defibrillation in the standard of care group is $180 \pm 180$ seconds. The expected time to defibrillation in the AECD group will be programmed into the device. Time to defibrillation will be coded continuously in both groups. Using a two-tailed t-test to compare time to defibrillation between the groups, a sample size of 12 patients per group affords $81 \%$ power at alpha $=0.05$. A total sample size of 40 patients per group will yield over $99 \%$ power for a two-tailed test at alpha $=0.05$.

\section{Statistical analysis}

The primary outcome will be time-to-defibrillation. Demographic and clinical categorical and continuous variables are compared for patients in the standard of care versus the AECD group at cardiac arrest. Baseline data will be expressed as mean \pm SD for continuous variables, and frequencies for categorical variables. Differences in baseline characteristics between the groups will be examined by the use of $\chi^{2}$ tests and two-sample t-tests, or, if assumptions are not met, by Fisher's Exact and Mann-Whitney tests for categorical and continuous variables, respectively. Time to defibrillation will be measured continuously in both the standard of care and the AECD group,

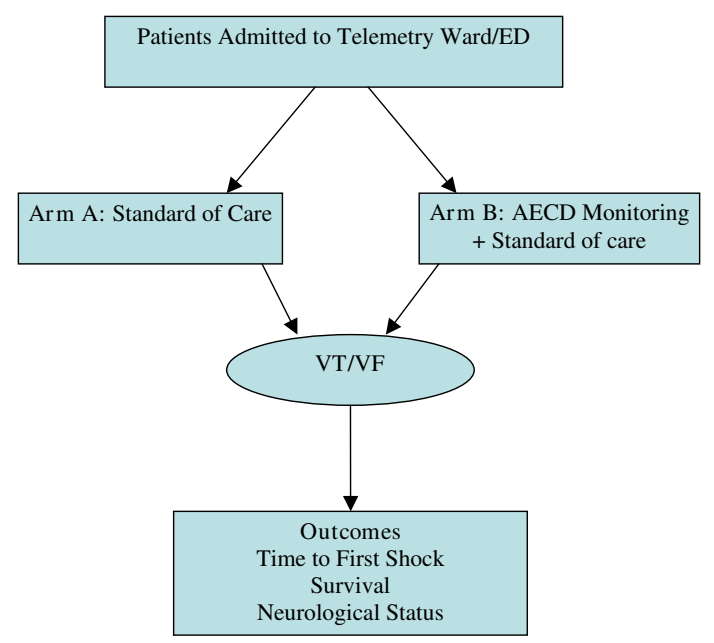

Figure I

Design of the AECD Trial. ED = emergency department; $\mathrm{AECD}=$ automated external cardioverter defibrillator; VT/ $\mathrm{VF}=$ ventricular tachycardia/ventricular fibrillation. 
Table 3: Demographic and Clinical Characteristics of Study Subjects

\begin{tabular}{|c|c|c|c|}
\hline & AECD $\mathrm{n}=95$ & Control $n=97$ & P Value \\
\hline Age \pm SD & $61.7 \pm 3.4$ & $62 \pm 3.4$ & 0.7 \\
\hline Gender & & & 0.98 \\
\hline Male & 92 (96.8\%) & 94 (96.9\%) & \\
\hline Female & $3(3.2 \%)$ & $3(3.1 \%)$ & \\
\hline Race & & & 0.15 \\
\hline Hispanic & $2(2.1 \%)$ & 0 & \\
\hline White & $42(44.2 \%)$ & $53(54.6 \%)$ & \\
\hline Black & $51(53.7 \%)$ & 44 (45.4\%) & \\
\hline History of HF & $25(26.3 \%)$ & $20(20.6 \%)$ & 0.39 \\
\hline New Diagnosis of HF & $6(6.3 \%)$ & $4(4.1 \%)$ & 0.53 \\
\hline Diabetes mellitus & $46(48.4 \%)$ & $35(36.1 \%)$ & 0.11 \\
\hline History of CAD & $35(36.8 \%)$ & $42(43.3 \%)$ & 0.38 \\
\hline Hypertension & $79(84 \%)$ & 74 (76.3\%) & 0.28 \\
\hline Hyperlipidemia & $55(57.9 \%)$ & $53(54.6 \%)$ & 0.66 \\
\hline EKG on admission & & & 0.62 \\
\hline NSR & $84(88.4 \%)$ & 81 (83.5\%) & \\
\hline Atrial fibrillation/flutter & $9(9.5 \%)$ & $11(11.3 \%)$ & \\
\hline SVT & 0 & $2(2.1 \%)$ & \\
\hline Other & $2(2.1 \%)$ & $3(3.1 \%)$ & \\
\hline
\end{tabular}

$\mathrm{SD}=$ standard deviation; $\mathrm{HF}=$ heart failure; $\mathrm{CAD}=$ coronary artery disease; NSR = normal sinus rhythm; SVT = supraventricular tachycardia

then compared using a two-tailed t-test. Assumptions will be checked and analyses will be adjusted accordingly.

In addition a multiple logistic model will be used to analyze the secondary endpoints (survival of cardiac arrest, survival to discharge), considering all the available variables. The total sample size will limit this analysis; however, an exploratory forward stepwise selection procedure will be used with a p-value of at least 0.10 for entry and 0.05 for removal. All pairwise interactions between AECD use and the other variables in the final stepwise model will be tested. A Hosmer-Lemeshow test will be used to test goodness of fit. Odds ratios will be presented with 95\% confidence intervals. Pearson residuals, deviance residuals, and influence statistics will be examined to assess model fit. Statistical analyses will be performed using SAS version 9.1.3.

Cerebral performance state (Table 2) will be measured using the cerebral performance categorization (CPC) scale and analyzed initially using $\chi^{2}$ tests. If cell counts warrant, the $2 \times 3$ contingency table will be analyzed using exact methods and the mean score statistics $(Q)$ will be used to compare cerebral performance status between the groups.

\section{Preliminary results}

One hundred and ninety two patients were recruited in the time period between $10 / 10 / 2006$ to $7 / 20 / 2007$. The demographic and clinical characteristics of the patients are presented in Table 3. Patients in the control and treatment arms had similar characteristics. The majority of patients (>90\%) in both arms were men, reflecting the demographics of the AVAMC. A total of 3,655 hours of telemetry data has been analyzed by the AECDs. The AECDs monitored ambulatory patients with normal sinus rhythm, sinus tachycardia, atrial fibrillation or flutter, supraventricular tachycardia, premature ventricular complexes and non-sustained ventricular tachycardia without inappropriately delivering a shock (Table 4). Of the 95 patients randomized to the AECD arm, total of ten patients had to be taken off the AECD, two due to adverse events, two due to anxiety, two due to skin irritation by the pads, and four due to alarming of the AECD, as the pads detached from the chest wall during their sleep.

Only one event of sustained VT occurred in the experimental arm, which was defibrillated by the AECD 17 seconds after programmed criteria were met (Figure 2). There are no events to report in the control arm. During the same time period mean time to shock for VT/VF cardiac arrest occurring outside the telemetry ward was $230 \pm 50$ seconds.

There were two false positive events in which, the shocks were delivered inappropriately. In the first case, the AECD delivered the shock when a patient was eating an apple.

Table 4: Frequency of Abnormal Rhythms Monitored by the AECD

\begin{tabular}{lc}
\hline Atrial Fibrillation/Flutter & $9(9.5 \%)$ \\
Supraventricular Tachycardia & $1(1.1 \%)$ \\
Premature Ventricular Complexes & $10(10.5 \%)$ \\
Non-Sustained Ventricular Tachycardia & $5(5.3 \%)$
\end{tabular}

$\mathrm{AECD}=$ automated external cardioverter defibrillator 


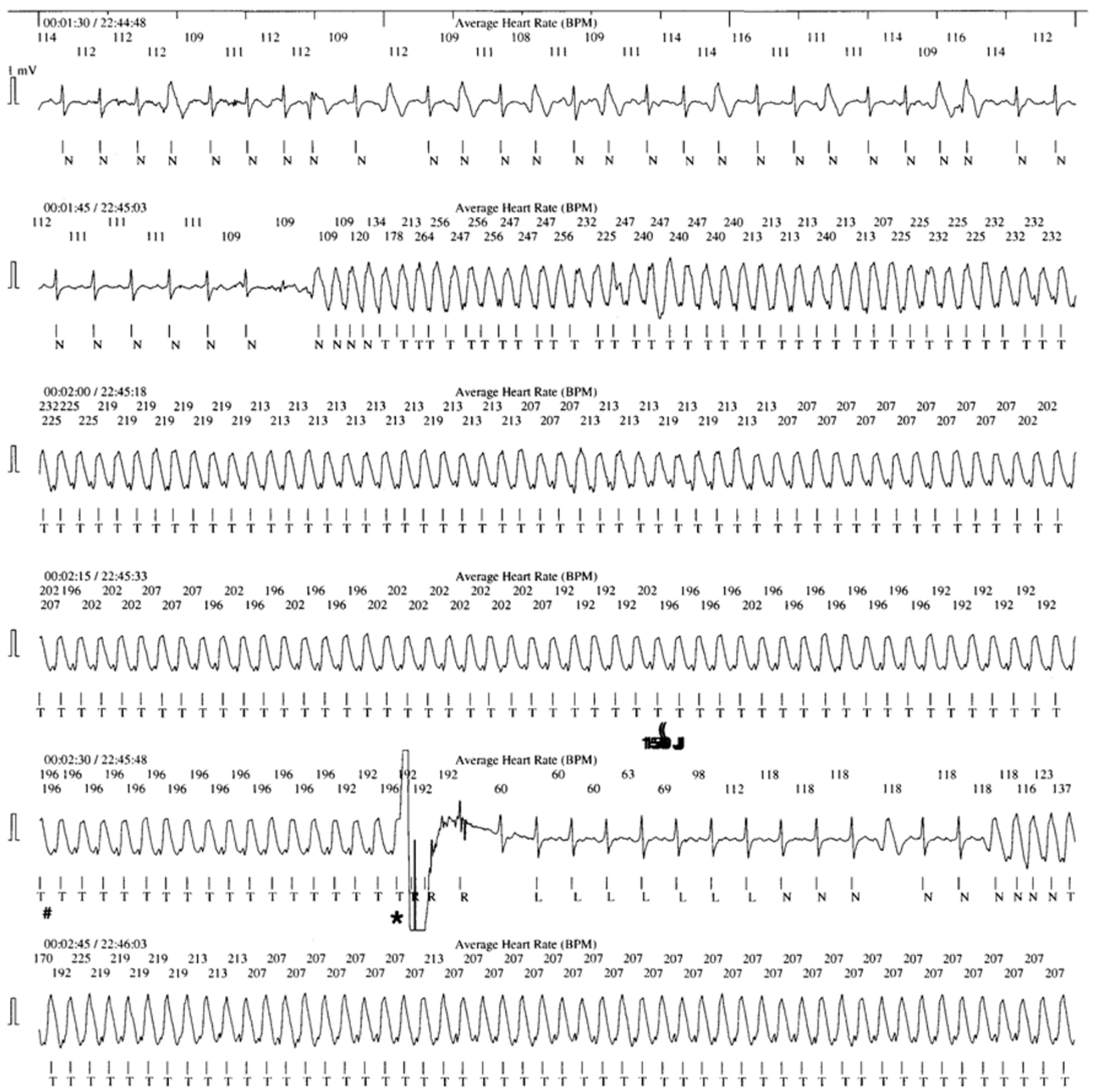

\section{Figure 2}

The AECD correctly recognized sustained monomorphic ventricular tachycardia and delivered a 150 Joule shock after 47 seconds. The rhythm is converted to sinus rhythm but reverts to ventricular tachycardia after 10 seconds. (= capacitor charging; \# $=$ capacitor charged; $*$ = therapy delivered to patient.

The AECD showed ventricular fibrillation at the same time the telemetry monitor showed sinus rhythm. This occurred in the setting of a reduced $\mathrm{R}$ wave amplitude on his 12-lead EKG, which was less than $0.5 \mathrm{mV}$. In the second case, discharge was the result of deliberate action of the patient. Neither patient had any other complications related to the delivery of the shocks. 


\section{Discussion}

In-hospital cardiac arrest remains a major health problem with high mortality rates.[2] Most important determinants of survival in both out-of-hospital and in-hospital cardiac arrest are early CPR and defibrillation.[1-3,10-12] Previously three clinical studies evaluated the application of AECD technology in hospitalized patients. [7-9] Mattioni et al. and Martinez-Rubio et al. both demonstrated high sensitivity and specificity for the AECD in the treatment of tachyarrhythmias.[7,8] The two studies demonstrated time-to-shock in the range of 14 to 22 seconds in patients admitted to the intensive care units and the cardiac electrophysiology laboratory.[7,8] In another study in patients with cardiovascular diagnoses admitted to the emergency department, a 94.4\% success rate, with a mean time-to-shock of 33.4 seconds for VT/VF arrhythmias was found.[9] These studies, while demonstrating effectiveness of AECDs in highly specialized settings, did not establish efficacy in an ambulatory group, where the possibility of inappropriate discharges are increased. In addition, none of the studies were randomized; there were no control groups with standard of care; and finally, none of the previous studies evaluated the impact of AECDs on survival and cerebral outcomes.

In the one arrest monitored by AECD, we showed a defibrillation time of 17 seconds. This is consistent with previous AECD trials. On the other hand, Chan and his coauthors reported that in $30 \%$ of in-hospital cardiac arrest, defibrillation is delayed using standard therapy, resulting in lower survival to discharge (22.2\% vs $39.3 \%$, $\mathrm{P}=<0.001$ ).[1] After hours (5 p.m. to 8 a.m.), weekends and unmonitored beds were predictors of delayed defibrillation, suggesting that manpower considerations were a critical determinant of response.[1,13] It would seem likely that routine use of automated strategies would help addressing these delays.[14] This idea is supported by findings that AEDs can improve outcomes in in-hospital cardiac arrest.[6]

Our trial thus far, has established the safety and practicality of continuous monitoring by AECDs in a high risk, ambulatory, inpatient population. Having established efficacy, continuation of the trial will allow a comparison of outcomes between the two strategies.

\section{Abbreviations}

AECD: automatic external cardioverter defibrillator; AED: automated external defibrillator; AVAMC: Atlanta Veterans Affairs Medical Center; CPC: cerebral performance categories; CPR: cardiopulmonary resuscitation; IRB: Institutional Review Board; NRCPR: National Registry of Cardiopulmonary Resuscitation; SD: standard deviation; VT/VF: pulseless ventricular tachycardia/ventricular fibrillation

\section{Competing interests}

Dr. Zafari reports receiving an investigator-initiated grant from Cardiac Science, Inc.

\section{References}

I. Chan PS, Krumholz HM, Nichol G, Nallamothu BK, American Heart Association National Registry of Cardiopulmonary Resuscitation Investigators: Delayed time to defibrillation after in-hospital cardiac arrest. N EnglJ Med 2008, 358:9-17.

2. Peberdy MA, Kaye W, Ornato JP, Larkin GL, Nadkarni V, Mancini ME, et al.: Cardiopulmonary resuscitation of adults in the hospital: a report of 14720 cardiac arrests from the national registry of cardiopulmonary resuscitation. Resuscitation 2003, 58:297-308.

3. Hazinski MF, Nadkarni VM, Hickey RW, O'Connor R, Becker LB, Zaritsky A: Editorial: major changes in the 2005 AHA Guidelines for CPR and ECC: reaching the tipping point for change. Circulation 2005, I I 2(suppl IV):IV-206-IV-2II.

4. Valenzuela TD, Roe DJ, Cretin S, Spaite DW, Larsen MP: Estimating effectiveness of cardiac arrest interventions. Circulation 1997, 96:3308-13.

5. Larsen MP, Eisenberg MS, Cummins RO, Hallstrom AP: Predicting survival from out-of-hospital cardiac arrest: a graphic model. Ann Emerg Med 1993, 22: I652-8.

6. Zafari AM, Zarter SK, Heggen V, Wilson P, Taylor RA, Reddy K, et al.: A program encouraging early defibrillation results in improved in-hospital resuscitation efficacy. J Am Coll Cardiol 2004, 44:846-52.

7. Mattioni TA, Nademanee K, Brodsky M, Fisher J, Riggio D, Allen B, et al.: Initial clinical experience with a fully automatic in-hospital external cardioverter defibrillator. PACE 1999, 22: 1648-55.

8. Martinez-Rubio A, Kanaan N, Borggrefe M, Block M, Makijarvi M, Fedele $F$, et al.: Advances for treating in-hospital cardiac arrest: safety and effectiveness of a new automatic external cardioverter-defibrillator. J Am Coll Cardiol 2003, 41:627-32.

9. Bento AM, Cardoso LF, Timerman S, Moretti MA, Peres ED, de Paiva EF, et al.: Preliminary in-hospital experience with a fully automatic external cardioverter-defibrillator. Resuscitation 2004, 63:11-6.

10. Valenzuela TD, Roe DJ, Nichol G, Clark LL, Spaite DW, Hardman RG: Outcomes of rapid defibrillation by security officers after cardiac arrest in casinos. N Engl J Med 2000, 343: I206-9.

II. Swedish Cardiac Arrest Registry: Factors modifying the effect of bystander cardiopulmonary resuscitation on survival in outof-hospital cardiac arrest patients in Sweden. Eur Heart J 200I, 22:5II-9.

12. White RD, Bunch TJ, Hankins DG: Evolution of a communitywide early defibrillation program experience over 13 years using police/fire personnel and paramedics as responders. Resuscitation 2005, 65:279-83.

13. Peberdy MA, Ornato JP, Larkin GL, Braithwaite RS, Kashner TM, Carey SM, et al:: Survival from in-hospital cardiac arrest during night and weekends. JAMA 2008, 299:785-92.

14. Saxon LA: Survival after tachyarrhythmic arrest-what are we waiting for? N EnglJ Med 2008, 358:77-79.

Publish with Bio Med Central and every scientist can read your work free of charge

"BioMed Central will be the most significant development for disseminating the results of biomedical research in our lifetime. "

Sir Paul Nurse, Cancer Research UK

Your research papers will be:

- available free of charge to the entire biomedical community

- peer reviewed and published immediately upon acceptance

- cited in PubMed and archived on PubMed Central

- yours - you keep the copyright 